\title{
Resources: Searching for Music for Performance (Scores and Parts)
}

\author{
Compiled by Erika Kirsch, McGill University
}

\section{Sources of information about sheet music}

- Emusicquest: The Music-in-Print Series (online version of the original print series)

- WorldCat

- Karlsruhe Virtual Catalog: http://www.ubka.uni-karlsruhe.de/kvk en.html

- Bonner Katalog: Verzeichnis reversgebundener musikalischer Aufführungsmateriale. Berlin: Walter De Gruyter, 2009.

- Library and Archives Canada; Library of Congress

$\circ$ http://www.collectionscanada.gc.ca/

○ http://www.loc.gov/

- Local catalogues of other institutions with music collections

- Oxford Music Online - useful for verifying opus numbers, correct titles

- Library of the institution where the composer taught

- IAMIC (International Association of Music Information Centres)

○ http://www.iamic.net/

- Religious or ethnic music information centres

$\circ \quad$ e.g. Milken Archive of American Jewish Music: http://www.milkenarchive.org/

- PROs (Performing Rights Organizations)

○ BMI - Broadcast Music, Inc.: http://www.bmi.com/

- ASCAP - American Society of Composers, Authors and Publishers http://www.ascap.com/index.aspx

- SOCAN - Society Of Composers, Authors and Music Publishers of Canada http://www.socan.ca/

- $\quad$ MPA (Music Publishers Association); CMPA (Canadian Music Publishers Association)

○ http://mpa.org/

○ http://www.musicpublishercanada.ca/

- Composer web pages, including those maintained by scholarly institutes

- Guides to repertoire by ensemble type

- Daniels, David. Orchestral Music: A Handbook. 4th ed. Lanham, MD: Scarecrow Press, 2005. Well-known for its instrumentation, publication, and timing information.

- Orchestral Music Online (online version of Daniels' book)

- Eaton, Quaintance. Opera Production: A Handbook. 2 vols. Minneapolis, MN: University of Minnesota Press, 1961-1974. Handy for opera orchestra instrumentations.

- Shrock, Dennis. Choral Repertoire. Oxford: Oxford University Press, 2009.

- Rehrig, William H. Heritage Encyclopedia of Band Music: Composers and Their Music. 3 vols. Westerville, OH: Integrity Press, 1991-1996.

- Manning, Lucy. Orchestral "Pops" Music: A Handbook. Lanham, MD: Scarecrow Press, 2009. 
- Schneider, Klaus. Lexikon Programmusik. 2 vols. Kassel: Bärenreiter, 1999-2000.

This resource can help you plan your program by suggesting themes.

- Chwialkowski, Jerzy. The Da Capo Catalog of Classical Music Compositions. New York: Da Capo Press, 1996.

This book breaks down large works into their various parts (by movement, by aria, etc.).

- Other ensemble librarians: Major Orchestra Librarians' Association (MOLA)

○ http://www.mola-inc.org

\section{Sources for acquiring sheet music}

- Petrucci Music Library, which includes the Choral Public Domain Library

○ http://imslp.org/wiki/Main_Page

- Educational Music Service, or any other local music dealer/supplier

○ http://www.emsmusic.com/

- Hal Leonard Corporation

○ http://www.halleonard.com/

- Reprint houses: Kalmus, Luck's, Broude Brothers

○ http://www.kalmus-music.com/

○ http://www.lucksmusic.com/

○ http://www.broude.us/

- Publisher websites

○ http://www.schott-music.com/

○ http://www.universaledition.com/

○ http://www.henle.de/

○ http://www.baerenreiter.com/

○ http://www.breitkopf.de/

○ http://www.edition-peters.com/home.php

- Rental agents

○ http://www.boosey.com/

○ http://www.schirmer.com/

○ http://www.eamdllc.com/ (European American Music Distributors)

- http://www.cpmusiclibrary.ca/ (Canadian agent for Oxford University Press, Edition Peters, and others)

- Canadian Music Centre

$\circ$ http://www.musiccentre.ca/

- Edwin A. Fleisher Collection of Orchestral Music

○ http://libwww.freelibrary.org/collections/collectionDetail.cfm?id=14

- Orchestra Musician's CD-ROM Library

- Sources for jazz charts

○ http://www.marinamusic.com/

○ http://www.uncjazzpress.com/

○ http://www.otterdist.com/ 